\title{
Building effective models from sparse but precise data: Application to an alloy cluster expansion model
}

\author{
Eric Cockayne \\ Ceramics Division, Materials Science and Engineering Laboratory, National Institute of Standards and Technology, Gaithersburg, \\ Maryland 20899-8520, USA \\ Axel van de Walle \\ Engineering \& Applied Science Division, California Institute of Technology, Pasadena, California 91125, USA
}

(Received 30 December 2009; published 27 January 2010)

\begin{abstract}
A common approach in computational science is to use a set of highly precise but expensive calculations to parameterize a model that allows less precise but more rapid calculations on larger-scale systems. Leastsquares fitting on a model that underfits the data is generally used for this purpose. For arbitrarily precise data free from statistic noise, e.g., ab initio calculations, we argue that it is more appropriate to begin with an ensemble of models that overfit the data. Within a Bayesian framework, a most likely model can be defined that incorporates physical knowledge, provides error estimates for systems not included in the fit, and reproduces the original data exactly. We apply this approach to obtain a cluster expansion model for the $\mathrm{CaZr}_{1-x} \mathrm{Ti}_{x} \mathrm{O}_{3}$ solid solution.
\end{abstract}

DOI: 10.1103/PhysRevB.81.012104

PACS number(s): 71.15.Nc, 02.50.Tt, 61.50.Lt, 71.15.Mb

A common approach in computational science is the use of a small number of highly precise but expensive calculations to generate data to fit the parameters of a less accurate but more computationally tractable "effective model" enabling larger-scale simulations. ${ }^{1,2}$ A typical example is the fit of a simplified energy model to accurate quantummechanical calculations. ${ }^{3-11}$ Although least-squares minimization is traditionally used for this purpose, it is not commonly recognized that this approach implicitly and incorrectly assumes that the uncertainty lies in the data rather than in the effective model.

Here we show that the fact that the model is less accurate than the data can be properly taken into account within a Bayesian ${ }^{12}$ framework where the "prior" probability distribution of the model parameters characterizes the range of physically plausible models. The model parameters are obtained by maximizing the "posterior" distribution provided by Bayes rule, given the accurate data and the prior. This approach enables a perfect fit to the input noiseless data while avoiding the usual artifacts of overfitting ${ }^{10}$ and enables the seamless inclusion of physical knowledge into the fitting procedure via the prior. Although Bayesian methods have a long history in the statistical sciences, (including recent interest in Bayesian learning techniques ${ }^{13,14}$ ), the unexpectedly well-behaved limit of completely noiseless data we report here has, to our knowledge, not been noted, perhaps because existing methods have historically been motivated by the need to fit noisy experimental data rather than noiseless calculated data. However, the latter setting clearly deserves more attention.

While our general theoretical approach should have broad applicability in numerous fields of computational sciences, in this paper, we focus on the specific but broadly applicable example of the construction of an efficient energy model for a crystalline alloy. ${ }^{3}$ This task has immediate applications to thermodynamic modeling of alloys and the determination of their phase diagrams, a crucial component of alloy design and optimization.
In this context, the accurate total-energy data are provided by $a b$ initio electronic-structure methods based upon densityfunctional theory, ${ }^{15,16}$ whose accuracy has been thoroughly validated in a wide range of solid-state systems. ${ }^{17}$ (Although such $a b$ initio calculations may not provide the exact quantum-mechanical result, they are precise in that they are virtually free of random errors, as numerical noise is well controlled in modern $a b$ initio software.) The effective model is a so-called cluster expansion (CE), ${ }^{3-11,18}$ that takes the form of a polynomial in occupation variables (described in detail below) indicating which atom lies on each lattice site. The unknown parameters of the CE to be determined are the coefficients of this polynomial. The $\mathrm{CE}$ has been previously shown ${ }^{18}$ to be able, in principle, to exactly represent any possible configurational dependence of the energy, provided that all terms the expansion are included, which unfortunately amounts to an infinite number of terms.

Typically, such CE models are created through a leastsquares fit to a database of $a b$ initio structural energies obtained using a CE truncated to a finite number of terms so that the number of input configurations is larger than the number of unknown parameters. This leads to a "truncation problem," where the terms to be retained in the model must be determined. Approaches for optimizing the truncation in this context have included the cross-validation (CV) score minimization, $, 310,19$ sometimes combined with regularization techniques..$^{20,21}$ Conventional Bayesian approaches have also been suggested, ${ }^{22,23}$ which do not restrict the number of unknown parameters to be bounded by the size of the database.

These approaches treat systematic errors (due to model truncation) and statistical errors (due to numerical noise in the data) on an equal footing without exploiting the knowledge that statistical errors are, in fact, negligible in this context. In the large sample limit, truncation selection methods would eventually "discover" that the statistical noise is zero but considerable improvements are possible if this known fact is explicitly taken into account from the start. 


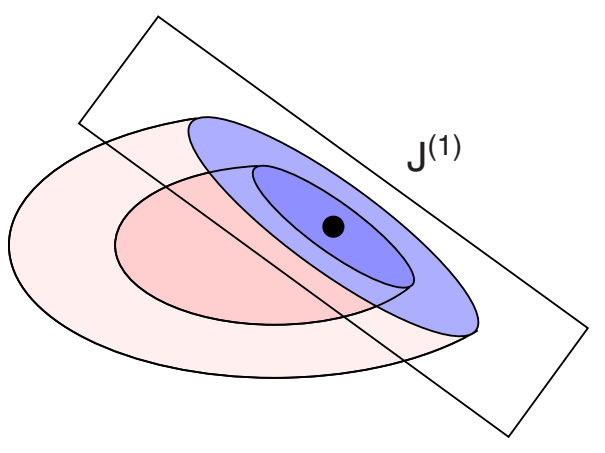

FIG. 1. (Color online) The ellipsoids schematically represent equiprobability surfaces of a many-dimensional prior probability distribution for $P^{0}(J)$. The plane represents the constraints on $J$ given by the results of an $a b$ initio total-energy calculation. The $(N-1)$-dimensional ellipsoid sliced by the plane gives an equiprobability surface for the posterior distribution of $P^{(1)}(J)$; the point marked $J^{(1)}$, represents the most likely solution for $J$.

In this paper, we avoid truncation problems by including many more terms in the effective model than the number of $a b$ initio calculations performed. Although the fitting problem is underdetermined, it can nonetheless be solved by using Bayesian inference with a physically based prior probability distribution for the model coefficients.

A configuration $i$ in a binary alloy is defined by the occupation of each site $k$ of a lattice by one of two species, indicated by a spinlike variable $s_{i k}= \pm 1$. Each configuration $i$ has an associated $a b$ initio energy $E_{i}$. These energies can be written in terms of effective cluster interactions, 6,18

$$
\sum_{\alpha} \xi_{i \alpha} J_{\alpha}=E_{i}
$$

where $\xi_{i \alpha}=\left\langle\Pi_{k \in \alpha} s_{i k}\right\rangle$ is the translationally and rotationally averaged multibody spin correlation for each symmetryindependent cluster $\alpha$ while $J_{\alpha}$ is the associated effective interaction parameter to be determined.

Bayes' theorem ${ }^{12}$ states that given a prior probability distribution $P^{(0)}(J)$ of the unknown parameter vector $J$ and one energy observation $E_{i}$, the posterior probability of $J$ given $E_{i}$ is

$$
P\left(J \mid E_{i}\right) \propto P\left(E_{i} \mid J\right) P^{(0)}(J) .
$$

Since $E_{i}$ is precisely known, the conditional probability reduces to a delta function,

$$
P\left(E_{i} \mid J\right)=\delta\left(\xi_{i} J-E_{i}\right),
$$

where $\xi_{i}$ denotes the row vector of all values of $\xi_{i \alpha}$ for a fixed $i$. As a result, $P\left(J \mid E_{i}\right)$ is trivially proportional to $\delta\left(\xi_{i} J-E_{i}\right) P^{(0)}(J)$. By induction, the posterior probability of $J$ based on all the energy information $E_{i}, i=1, \ldots, n$ is

$$
P^{(n)}(J) \propto \prod_{i=1}^{n} \delta\left(\xi_{i} J-E_{i}\right) P^{(0)}(J) .
$$

The difference between prior and posterior probabilities is represented geometrically in Fig. 1. Each new data point selects a cross section of the prior distribution corresponding
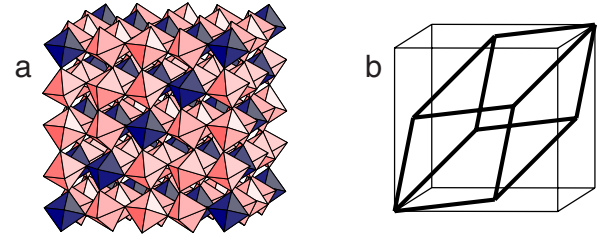

FIG. 2. (Color online) (a) Representative CZT structure; (b) common 80-atom supercell for CZT energy calculations highlighted in bold.

to sets of parameter values that agree perfectly with this data point. Within the intersection of these cross sections, each point has a different posterior probability that is dictated by the prior. The most likely model parameters $J^{(n)}$ can be determined by maximizing $P^{(n)}(J)$ (see key methodological details at end). This approach selects a unique solution from an otherwise underdetermined system of equations, based on the "physical" information provided by the prior.

The width of the posterior provides a measure of the uncertainty remaining in the fitted parameter after the data has been incorporated, which can be used, for instance, to access the accuracy of predicted energies for any structure not included in the fit. For a Gaussian prior, the posterior is Gaussian as well and the most likely parameter values are also the expected parameter values, which implies, given the linearity of the cluster expansion, that the predicted energies from the $\mathrm{CE}$ model will also be expected values.

The above procedure was applied to model total energies in the $\mathrm{CaZr}_{1-x} \mathrm{Ti}_{x} \mathrm{O}_{3}(\mathrm{CZT})$ system, a perovskite solid solution with tilted oxygen octahedra. ${ }^{24}$ The structures studied were constrained to a common 80 -atom supercell shown in Fig. 2, which has 16 perovskite " $B$ " sites that contain either $\mathrm{Zr}$ or Ti. Local density-functional theory calculations were performed on specific CZT configurations using the VASP (Ref. 25) code and ultrasoft pseudopotentials, ${ }^{26}$ with semicore $p$ electrons treated as valence electrons for $\mathrm{Ca}, \mathrm{Zr}$, and Ti. A $375 \mathrm{eV}$ plane-wave energy cutoff and a $1500 \mathrm{eV}$ cutoff for augmentation charges were used. The $k$-point mesh was equivalent to an $8 \times 8 \times 8$ Monkhorst-Pack grid for a primitive perovskite cell. The number of symmetrically distinct possible arrangements of $\mathrm{Zr}$ and $\mathrm{Ti}$ and the number of terms in the full cluster expansion are both 2386; all terms are retained in the fitting.

Based on simple physical considerations, the following Gaussian prior was selected:

$$
P^{(0)}(J)=\prod_{\alpha}\left(\sqrt{2 \pi} w_{\alpha}\right)^{-1} \exp \left[\left(-J_{\alpha}\right)^{2} /\left(2 w_{\alpha}^{2}\right)\right]
$$

with $w_{\alpha}=A b^{n} \alpha \prod_{\{i, j\} \subset \alpha}\left(r_{i j} / a\right)^{-2}$ (a, the primitive perovskite lattice parameter, $\approx 4 \AA \AA$ ). Clusters with more sites $n_{\alpha}$ are expected to have smaller coefficients (i.e., $b<1$ ), and clusters with pairs $\{i, j\}$ of atoms at larger separations $r_{i j}$ are expected to have smaller coefficients. The exponent of -2 is motivated by the observation that $\mathrm{Zr}$ and $\mathrm{Ti}$ have the same charge so interactions cannot be mediated by differences in monopole coupling, leaving only dipolar leading terms. The choice of a Gaussian prior is not only computationally convenient but also reflects the fact that the effective interaction 


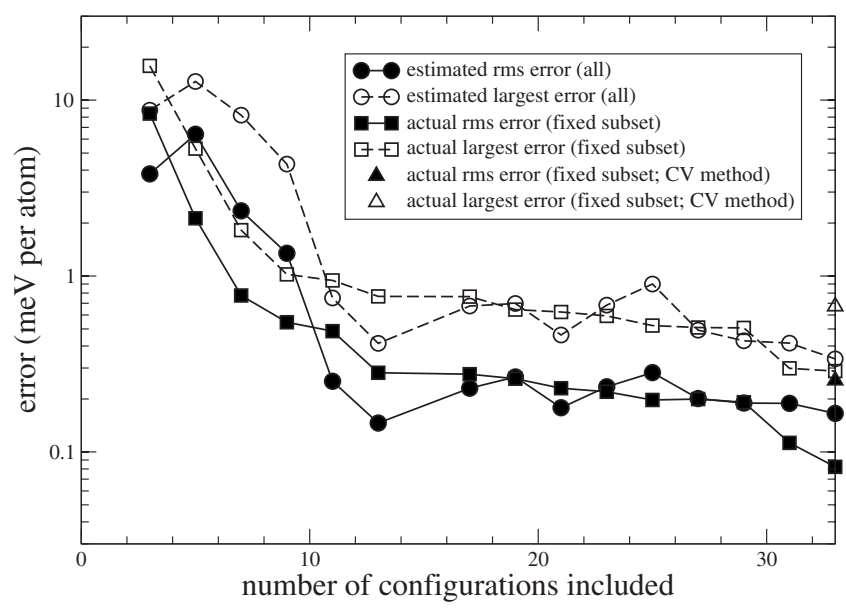

FIG. 3. (Circles) Convergence of the mean and maximum predicted errors on energies of configurations not included in the fit, as a function of the number of configurations included in fit. (Squares) Convergence of actual errors for a selected subset of 37 structures not included in the fit. (Triangles) Predictive ability of CV method.

between a given set of atoms is the result of large number of small (a priori random) contributions. A central limit argument then motivates the fact that the effective interactions should, a priori, have a Gaussian distribution.

Although, in a traditional Bayesian setting, the parameters $A$ and $b$ are user specified, we devised a technique to optimize them (see key methodological details), thus making the method free of adjustable parameters. In essence, we view the posterior as a statistical quantity whose sampling properties (as benchmarked by cross validation ${ }^{10}$ ) can be optimized by adjusting the prior.

Our Bayesian approach is embedded in an iterative scheme where, at each step, a most likely solution $J$ is found and error estimates for the other configurations not included in the fit are calculated using the posterior. The configuration with the highest estimated error is then added to the fit, after its ab initio total energy is calculated (see key methodological details). This procedure is repeated until a sufficient predictive accuracy has been reached, as illustrated in Fig. 3, which compares the estimated errors with the actual errors on a fixed subset of 37 selected structures not used in the fitting procedure. One sees: (1) the fit rapidly improves at first, as the dominant terms are determined, followed by a slower improvement; (2) the estimated errors fluctuate but generally match the true errors to within a factor of 2 ; and (3) the predictive errors are two to three times smaller than those for the cross-validation approach.

The proposed method offer one very important advantage for thermodynamic applications, such as phase-diagram calculations,${ }^{10}$ where it is crucial that the lowest-lying energies have the proper order and energy differences. The conventional least-squares fitting procedure does not guarantee this, even if these states were included in the fitting procedure. By reproducing the energies of all states included in the fit exactly, our procedure entirely avoids this problem.

For calculation efficiency purposes, one may truncate the terms to be retained in the solution to those whose $J_{\alpha}$ exceeds some small threshold value, with little effect on the results (as suggested in Ref. 20 in a related context).

While the need to specify a prior in Bayesian methods is often criticized, it should be realized that a conventional least-squares fit is not free of a priori assumptions either. In the cluster expansion example, a conventional fit with a userspecified truncation distance amounts to a prior which is uninformative (flat) for the included interactions coefficients but entirely concentrated at zero for the excluded coefficients. This incorporates physical knowledge into the problem but with a complete certainty that far exceeds what a researcher could plausibly know. A smoother prior which gradually concentrates the probability toward zero coefficient values as the range of interaction increases appears a more appropriate description of a priori information.

If the data does contain some error (for instance, significant numerical noise), then the delta function in Eq. (1) must be replaced by a smooth density and a conventional Bayesian methodology would result (as in Refs. 22 and 23). If that density is Gaussian, then one recovers a so-called ridge regression or Tikhonov regularization, ${ }^{27}$ a penalized leastsquares estimator, which has been previously used in the context of CE construction..$^{20,21}$

While our procedure generates a model that is uniquely determined from the input data, the physical knowledge incorporated is minimal, and priors that incorporate more physical knowledge would likely yield superior models with even better predictive ability. For example, one could choose a prior where the values of terms related by pseudosymmetry relationships are correlated ${ }^{23}$ or priors where the multibody terms include angular dependence. Formally quantifying this method's discriminating power as a function of the prior represents a fruitful avenue of future investigation.

The construction of multibody interatomic force fields based on quantum-mechanical data ${ }^{28,29}$ represents an important possible application of the methodology proposed here. It differs from the alloy problem in that the energy is a function of a continuous set of distance variables, rather than a discrete set of spacings on a lattice. While force fields are often selected to be nonlinear in the unknown parameters, energy models that are linear in the parameters (such as splines) are probably preferable in our context, as they make the interaction optimization problem numerically stable and efficient. The lack of physical basis for spline functions is alleviated by the possibility of including many more parameters than data points and the ability to favor physically plausible interaction shapes via the prior. Such an approach could lead to successful force-field models, with defined uncertainties and with broad applications in computational physics (see Ref. 30 for recent work on the Bayesian approach to modeling of force fields).

Key methodological details. With a zero-mean Gaussian prior for the vector $J$ containing all interactions $J_{\alpha}$, the posterior maximum is given by $J^{(n)}=W \xi^{T}\left(\xi W \xi^{T}\right)^{-1} E$, where $W$ denotes the variance-covariance matrix of the prior while $\xi$ denotes the matrix with elements $\xi_{i \alpha}$ and $E$ denotes the vector of all $n$ known structural energies $E_{i}$. An equivalent approach is to perform the changes in variables $\xi^{\prime}=\xi W^{1 / 2}$ and $J^{\prime(n)}=W^{-1 / 2} J^{(n)}$ (where we use the symmetric square-root matrix) and find the minimum Euclidean norm solution $J^{\prime(n)}$ to 
$\xi^{\prime} J^{\prime(n)}=E$ using, for instance, standard singular-value decomposition techniques.

The expected energy of any additional configuration $i=n+1$ (with correlations equal to the row vector $\xi_{(n+1)}$ ) is given by $\xi_{(n+1)} J^{(n)}$. The root-mean-square error in this energy is simply given by $\left|\operatorname{Pr}_{\perp}^{\prime(n)} \xi_{n+1}^{\prime}\right|$, where $\operatorname{Pr}_{\perp}^{\prime(n)}$ is a projector in the prime space that simultaneously projects $\xi_{n+1}^{\prime}$ orthogonal to all $\xi_{i \leq n}^{\prime}$.

The parameters of the prior are optimized as follows. If all terms $w_{\alpha}$ in a Gaussian prior are scaled by the same value $x$, then (1) $J^{(n)}$ remains unchanged and (2) all predicted errors are scaled by $x$. A self-consistent value for $x$ can therefore be obtained by scaling the mean-square estimated errors to equal the actual errors. In an analogous way to the leave-oneout cross-validation method, ${ }^{10}$ one can take the set of struc- tures used to obtain $J^{(n)}$, omit each single structure $i$ one at a time, and calculate the estimated and actual errors for $E_{i}$ based on fitting the model to all the other structures. The value $b$ is optimized by minimizing the mean-square crossvalidation error. In our example, as more structures are included, the value of $b$ converges, which indicates that the procedure is self-consistent.

A.v.d.W. was supported by the U.S. National Science Foundation via TeraGrid resources at NCSA and SDSC under Grant No. TG-DMR050013N. This material is based upon work supported by the Department of Energy National Nuclear Security Administration under Award No. DE-FC5208NA28613 and National Energy Research Initiative Consortium (NERI-C) under Award No. DE-FG07-07ID14893.
${ }^{1}$ S. Yip, Handbook of Materials Modeling (Springer, The Netherlands, 2005).

${ }^{2}$ G. Olson, Science 277, 1237 (1997).

${ }^{3}$ G. L. W. Hart, V. Blum, M. J. Walorski, and A. Zunger, Nature Mater. 4, 391 (2005).

${ }^{4}$ D. de Fontaine, Solid State Phys. 47, 33 (1994).

${ }^{5}$ F. Ducastelle, Order and Phase Stability in Alloys (Elsevier Science, New York, 1991).

${ }^{6}$ J. W. D. Connolly and A. R. Williams, Phys. Rev. B 27, 5169 (1983).

${ }^{7}$ M. Asta, V. Ozolins, and C. Woodward, JOM 53, 16 (2001).

${ }^{8}$ G. Ceder, A. van der Ven, C. Marianetti, and D. Morgan, Modell. Simul. Mater. Sci. Eng. 8, 311 (2000).

${ }^{9}$ A. Zunger, in Statics and Dynamics of Alloy Phase Transformation, NATO Advanced Studies Institute, Series B: Physics, Vol. 319, edited by P. E. Turchi and A. Gonis (Plenum, New York, 1994), p. 361.

${ }^{10}$ A. van de Walle and G. Ceder, J. Phase Equilib. 23, 348 (2002).

${ }^{11}$ A. V. Ruban and I. A. Abrikosov, Rep. Prog. Phys. 71, 046501 (2008).

${ }^{12}$ E. T. Jaynes, Probability Theory: The Logic of Science (Cambridge University Press, Cambridge, UK, 2003), Vol. 1.

${ }^{13}$ M. E. Tipping, J. Mach. Learn. Res. 1, 211 (2001).

${ }^{14}$ M. A. T. Figueiredo and A. K. Jain, IEEE Trans. Pattern Anal. Mach. Intell. 24, 381 (2002).

${ }^{15}$ R. O. Jones and O. Gunnarsson, Rev. Mod. Phys. 61, 689
(1989).

${ }^{16}$ M. C. Payne, M. P. Teter, D. C. Allan, T. A. Arias, and J. D. Joannopoulos, Rev. Mod. Phys. 64, 1045 (1992).

${ }^{17}$ J. P. Perdew, J. A. Chevary, S. H. Vosko, K. A. Jackson, M. R. Pederson, D. J. Singh, and C. Fiolhais, Phys. Rev. B 46, 6671 (1992).

${ }^{18}$ J. M. Sanchez, F. Ducastelle, and D. Gratias, Physica A 128, 334 (1984).

${ }^{19}$ M. H. F. Sluiter, Y. Watanabe, D. de Fontaine, and Y. Kawazoe, Phys. Rev. B 53, 6137 (1996).

${ }^{20}$ A. Díaz-Ortiz, H. Dosch, and R. Drautz, J. Phys.: Condens. Matter 19, 406206 (2007).

${ }^{21}$ A. Zunger, L. G. Wang, G. L. W. Hart, and M. Sanati, Modell. Simul. Mater. Sci. Eng. 10, 685 (2002).

${ }^{22}$ A. P. J. Jansen and C. Popa, Phys. Rev. B 78, 085404 (2008).

${ }^{23}$ T. Mueller and G. Ceder, Phys. Rev. B 80, 024103 (2009).

${ }^{24}$ I. Levin, E. Cockayne, M. W. Lufaso, J. C. Woicik, and J. E. Maslar, Chem. Mater. 18, 854 (2006).

${ }^{25}$ G. Kresse and J. Furthmuller, Phys. Rev. B 54, 11169 (1996).

${ }^{26}$ D. Vanderbilt, Phys. Rev. B 41, 7892 (1990).

${ }^{27}$ A. N. Tikhonov, Dokl. Akad. Nauk SSSR 39, 195 (1943).

${ }^{28}$ V. Sundararaghavan and N. Zabaras, Phys. Rev. B 77, 064101 (2008).

${ }^{29}$ J. D. Gale and A. L. Rohl, Mol. Simul. 29, 291 (2003).

${ }^{30}$ A. P. Bartók, M. C. Payne, R. Kondor, and G. Csányi, arXiv:0910.1019 (unpublished). 